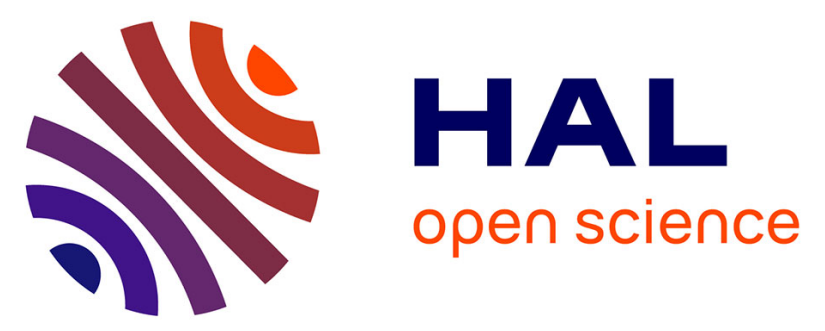

\title{
Patterning linear and nonlinear optical properties of photosensitive glasses by femtosecond structured light
}

Konstantin Mishchik, Yannick Petit, Etienne Brasselet, Arnaud Royon, Thierry Cardinal, Lionel Canioni

\section{- To cite this version:}

Konstantin Mishchik, Yannick Petit, Etienne Brasselet, Arnaud Royon, Thierry Cardinal, et al.. Patterning linear and nonlinear optical properties of photosensitive glasses by femtosecond structured light. Optics Letters, 2015, 40 (2), pp.201-204. 10.1364/OL.40.000201 . hal-01104961

\section{HAL Id: hal-01104961 \\ https://hal.science/hal-01104961}

Submitted on 26 Feb 2015

HAL is a multi-disciplinary open access archive for the deposit and dissemination of scientific research documents, whether they are published or not. The documents may come from teaching and research institutions in France or abroad, or from public or private research centers.
L'archive ouverte pluridisciplinaire HAL, est destinée au dépôt et à la diffusion de documents scientifiques de niveau recherche, publiés ou non, émanant des établissements d'enseignement et de recherche français ou étrangers, des laboratoires publics ou privés. 


\title{
Patterning linear and nonlinear optical properties of photosensitive glasses by femtosecond structured light
}

\author{
Konstantin Mishchik, ${ }^{1,2}$ Yannick Petit, ${ }^{1,2,3,4, *}$ Etienne Brasselet, ${ }^{1,2}$ Arnaud Royon, ${ }^{1,2}$ \\ Thierry Cardinal, ${ }^{3,4}$ and Lionel Canioni ${ }^{1,2}$ \\ ${ }^{1}$ University of Bordeaux, LOMA, UMR 5798, F-33400 Talence, France \\ ${ }^{2}$ CNRS, LOMA, UMR 5798, F-33400 Talence, France \\ ${ }^{3}$ CNRS, ICMCB, UPR 9048, F-33608 Pessac, France \\ ${ }^{4}$ University of Bordeaux, ICMCB, UPR 9048, F-33400 Pessac, France \\ *Corresponding author: yannick.petit@u-bordeaux.fr
}

\begin{abstract}
We report on structured light-induced femtosecond direct laser writing (DLW) under tight focusing in non-commercial silver-containing zinc phosphate glass, which leads to original patterns of fluorescent silver clusters. These fluorescence topologies show unique features of frustrated diffusion of charged species, giving rise to distorted silver cluster spatial distributions. Fluorescence and second harmonic generation correlative microscopy demonstrate the realization of structured light-induced direct laser poling, resulting from a laser-induced permanent and stable electric field buried inside the modified glass. Thus, structured light-induced DLW remarkably enables both linear and nonlinear patterning. This work highlights the interest of optical phase engineering to obtain nontrivial beam profiles and subsequent photo-induced patterns that cannot be reached under Gaussian beam irradiation.

OCIS codes: (140.3390) Laser materials processing; (260.6042) Singular optics; (190.4180) Multiphoton processes;
\end{abstract} (110.4234) Multispectral and hyperspectral imaging; (190.4400) Nonlinear optics, materials.

During the last decade, the versatile and cost-limited technique of 3D femtosecond (fs) direct laser writing (DLW) has led to huge progress [1]. Indeed, new laserinduced structures and functionalities have been obtained, especially because of two complementary fields of research, namely the engineering of complex light [2] and the development of tailored materials [3].

DLW with tightly focused Gaussian TEM $_{00}$ beams can result in locally isotropic material modifications, but also in highly anisotropic structures such as nanogratings [4]]. However, the bell-shaped nature of the Gaussian beam limits the range of accessible topologies. Such limitation was first partially overcome by considering structured light, referring to electromagnetic fields endowed with phase or polarization singularities (also called vortices). This appeared to be a powerful tool to carry out original vortex-induced processing of optical materials, by enabling surface ablations with donut-shaped topologies $[5,6]$ or the retrieval of the polarization distributions of complex light [7]. Moreover, vortex-assisted DLW, which derives from the stimulated emission depletion (STED) approach [8], has also led to super-resolution DLW $[9,10]$. Since structured light can be engineered on demand with spatial light modulators, with setups based on anisotropic crystals, or with specially designed plates engineered by DLW [11,12], it opens a large range of accessible shapes. However, vortex-inducted DLW has only led to the pattering of linear optical properties.

Concerning developments of optical materials, the photosensitizing with silver ions appears promising [3]. Indeed, the silver-doping of gallo-phosphate glasses highly improves the manufacturing quality of fs laserinduced nanogratings [13]. With noncommercial silverdoped phosphate glasses, it has been shown that the interaction of a high repetition rate fs pulse train gives rise to an original spatial distribution of photo-induced silver clusters below the diffraction limit [14], leading to fluorescent patterns [15], as well as second- [16-18] and third-order [19] nonlinear optical properties. Thus, such developments in silver-doped glasses appear relevant for new patterning with structured light, of both linear and nonlinear optical properties, the latter remaining an open challenge.

In this Letter, we report on structured light-induced fs DLW of silver-containing phosphate glasses. Complex fluorescent patterns that cannot be obtained using Gaussian beams are created. In particular, we demonstrate the creation of (i) nested fluorescent rings using LaguerreGauss-like beams, and (ii) multi-ring fluorescent structures using Hermite-Gauss-like beams. Noteworthy, the latter case gives access to inner spatial structuring with estimated dimensions down to $210 \pm 30 \mathrm{~nm}$. Moreover, effective second harmonic generation (SHG) is reported, highlighting the relationship between the laser-induced linear and nonlinear optical properties. Finally, we discuss our results according to mechanisms at play during DLW in these tailored glasses $[3,17]$.

Our tailored glass is a silver-containing zinc phosphate glass with a $5 \% \mathrm{Ag}_{2} \mathrm{O}$ concentration (mol. \%), prepared by a standard glass melting method, cut to $1 \mathrm{~mm}$ thick sample and polished to optical quality [20]. DLW was performed with a Ti:Sa regenerative laser amplifier (RegA 9000 from Coherent, up to $1 \mathrm{~W}, 250 \mathrm{kHz}, 60 \mathrm{fs}$ at $800 \mathrm{~nm}$ ). The irradiation duration and transmitted irradiance were controlled by an acousto-optic modulator, enabling the accumulation of $N=10^{5}-10^{6}$ pulses with energies from 50 to $150 \mathrm{~nJ}$. The positioning and displacement of the sample were performed with a high-precision 
3D translation stage (XMS-50 stages, Micro-Contrôle). Irradiations were carried out by focusing laser pulses with a microscope objective (Mitutoyo, APO PLAN VIS, $50 \times$ NA 0.55$)$. Pulse duration $($ FHWM) at the focus in the sample was $145 \mathrm{fs}$ (Gaussian beams) and $200 \mathrm{fs}$ (structured beams).

To perform structured light-induced DLW, the spatial engineering of the writing light field was set both in phase and amplitude [2]. We used a c-cut $8 \mathrm{~mm}$-thick uniaxial calcite crystal slab (optical axis oriented along the propagation direction, $n_{o}=1.649, n_{e}=1.482$ at $800 \mathrm{~nm}$ ), two quarter-wave plates, and a polarization beam splitter (Fig. 1). In a first configuration, the incident linearly polarized Gaussian field $\mathbf{E}_{0}$ [Fig. 2(a)] was transformed into a circularly polarized light field by the use of a $\lambda / 4$ plate, then focused with the lens $\mathrm{L} 1(f=50 \mathrm{~mm})$ through the calcite crystal, and re-collimated with the second lens L2 $(f=50 \mathrm{~mm})$. After the second $\lambda / 4$ plate, the inhomogeneously polarized light field was separated into two orthogonal linearly polarized components, thanks to a polarizing beam splitter (PBS). With our settings (Fig. 1), the component $\mathbf{E}_{\perp}$ led to a charge-2 optical vortex beam [21], whose intensity distribution is shown in Fig. 2(b). In a second configuration, both $\lambda / 4$ plates were removed. The incident Gaussian beam led to a four-lobe intensity pattern for the component $\mathbf{E}_{\perp}$ [22], as shown in Fig. 2(c).

The resulting linear and nonlinear optical responses were observed with fluorescence and SHG microscopy. Fluorescence imaging of the photo-modified areas was performed with a confocal microscope with an excitation wavelength at $405 \mathrm{~nm}$, for an emission in the $430-480 \mathrm{~nm}$ range (Leica, SP8, objective $63 \times$, NA 1.3 , and lateral resolution of 250-290 $\mathrm{nm}$ ). Nonlinear SHG imaging was performed with a homemade microscope setup (typical lateral resolution of $300-350 \mathrm{~nm}$ ), as detailed elsewhere [18].

DLW has been carried out with Gaussian, charge-2 optical vortex and four-lobe laser beams, whose intensity profiles before the focusing objective and in the laserwriting focal plane are shown in Figs. 2(a)-2(c) and in Figs. 2(d)-2(f), respectively. Gaussian-beam based DLW leads to the creation of a fluorescent hollow pipe [Figs. 2(g) and 2(j)], composed of fluorescent silver clusters, as reported elsewhere [14]. Previous works had proposed the following interpretation of the mechanisms at play:

- First, DLW activates four-photon ionization [14], generating mobile photoelectrons, mostly at the center of the focused spot where the intensity is higher. This

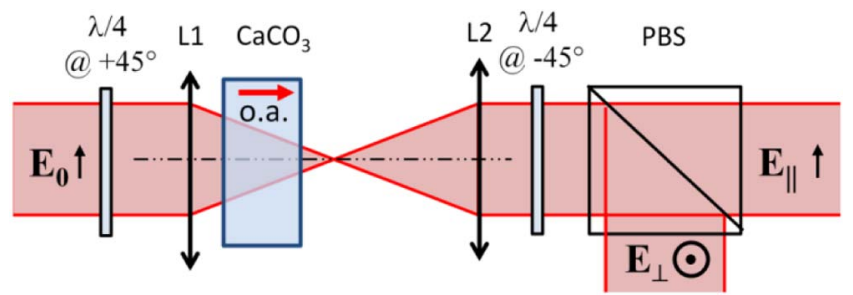

Fig. 1. Structured light generator from an incident linearly polarized Gaussian beam. Two settings, with or without the two $\lambda / 4$ plates, are used. Structured light-induced DLW is performed with the $\mathbf{E}_{\perp}$ polarized component.
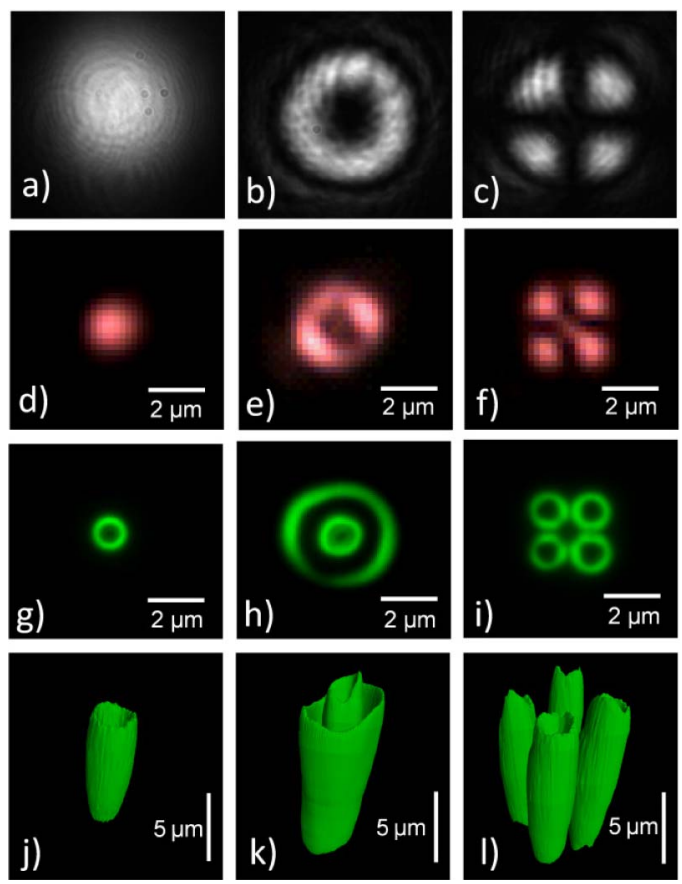

Fig. 2. Intensity profiles of the incident and focused beams, respectively: (a), (d) Gaussian; (b), (e) charge-2 vortex; and (c), (f) four-lobe. Related fluorescence patterns under top view and 3D view, respectively: (g), (j) single-wall hollow pipe; (h), (k) double-wall hollow pipe; and (i), (l) four single-wall hollow pipes.

is followed by the photoelectron diffusion toward the periphery of the intensity profile of the laser beam. Such multi-pulse DLW may also promote band gap excitation of photoelectrons from laser-induced species [23].

- Second, such diffusion of charged species followed by their trapping at the periphery of the focused laser beam leads to a space charge separation and, consequently, to the creation of an intense buried electric field (estimated to up to $8 \times 10^{8} \mathrm{~V} \cdot \mathrm{m}^{-1}$ [18]). Such an electric field is remarkably stable under thermal constraint, as long as the temperature remains below the glass transition temperature $\left(T_{g} \sim 385^{\circ} \mathrm{C}\right)$, while it completely vanishes above $T_{g}$ [18]. Moreover, it couples with the third-order nonlinear response of the glass, giving rise to an electric field induced second-harmonic generation (EFISHG) with $\chi_{\text {eff }}^{(2)} \sim 0.6 \mathrm{pm} \cdot \mathrm{V}^{-1}[\underline{18}]$, corresponding to efficient direct laser poling. From fluorescence and SHG correlative microscopy, both the silver cluster stabilization and the creation of a space charge separation had been monitored. In particular, the EFISHG response was proved to appear prior to the fluorescence emission, meaning that the creation and stabilization of the silver cluster is a consequence of the creation of the static electric field [17].

- Third, fluorescence and SHG correlative microscopy studies had evidenced that the silver clusters get stabilized where the electric potential modifications (resulting from the frozen space charge separation) locally lead to more favorable reduction-oxidation conditions.

For Gaussian beams [Fig. 2(d)], the single-wall hollow pipe shape is independent of the polarization of the 
incident laser, suggesting that such shape is driven only by the laser intensity profile. In contrast, DLW using structured light creates original fluorescent patterns. Indeed, a charge-2 vortex beam [Fig. 2(e)] induces a fluorescent pattern that corresponds to a double-wall hollow pipe [Figs. 2(h) and 2(k)], whereas a four-lobe beam [Fig. 2(f)] creates four single-wall hollow pipes [Figs. 2(i) and 2(1)]. These observations corroborate an intensitydriven mechanism, where phase plays no significant role in the multiphoton ionization step that releases the initial free electrons. Moreover, DLW is independent of the sign of the topological charge of the charge-2 vortex. Regarding the four-lobe beam, the dark cross is associated to $\pi$-phase steps, though the four bright spots produced identical fluorescent hollow pipes, showing again the independence of DLW with respect to phase. Still, the spatial distribution of the phase of the writing beam plays a crucial role, as it allows controlling the intensity profile of the incident light field, thereby the lightinduced patterns.

The dependence of structured light-induced patterns on the irradiation parameters has also been studied. For $10^{5}$ pulses, DLW thresholds of fluorescence are well below the typical self-focusing power $P_{\mathrm{cr}} \sim 6 \mathrm{MW}$, and the related energies are estimated to be $E_{p}=23,100$, and $67 \mathrm{~nJ}$ for Gaussian, charge-2 vortex, and four-lobe beams, respectively, locally corresponding to $3.8,4.3$, and $3.7 \mathrm{TW} / \mathrm{cm}^{2}$. The increase of the number of pulses or of the incident irradiance mainly leads to patterns with larger dimensions and brighter fluorescence emission [Fig. 3(a)]. However, it also reveals that significant spatial distortions may occur. The four-ring pattern obtained with the four-lobe beam at low intensity turns into a four-leaf clover pattern as the irradiance is increased
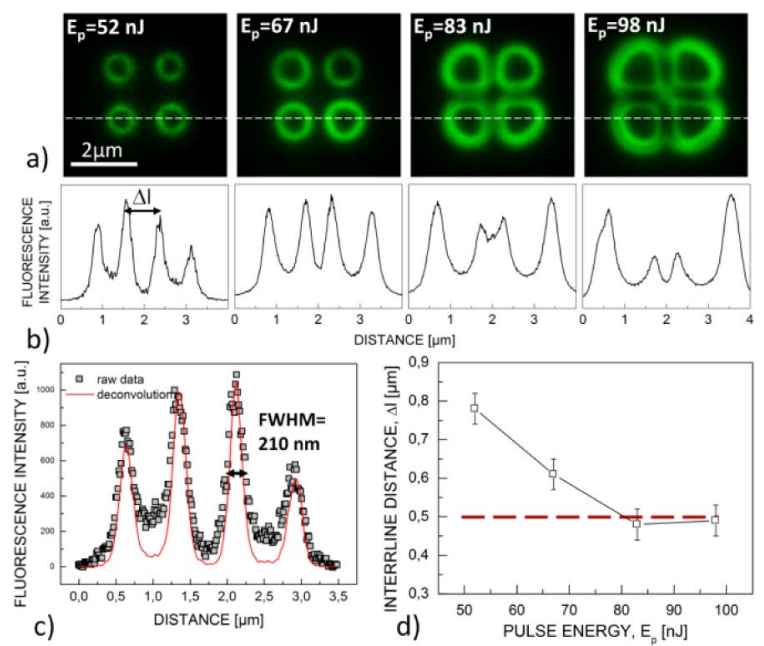

Fig. 3. Four single-ring fluorescent pattern versus incident irradiance. Nonmerging individual structures at high irradiances, underlying the electrically charged nature of the diffusive species, and highlighting their repulsion behavior: (a), (b) 2D top views and related horizontal profiles versus irradiance; (c) profile along the white dashed line for $E_{p}=52 \mathrm{~nJ}$ pulse energy and $N=10^{6}$ pulses, with four fluorescent rings with an estimated thickness down to $210 \mathrm{~nm}$; and (d) inter-distance $\Delta l$ between two fluorescent rings, showing a plateau around $500 \mathrm{~nm}$, which we attribute to the balance between diffusion and repulsion forces.
[Fig. 3(a), $E_{p}=98 \mathrm{~nJ}, 10^{6}$ pulses]. In such a distorted case, the four single-wall hollow pipes cannot be considered as independent anymore because their diameters become large enough to repel each other. The photoelectrons' diffusion of each single-wall hollow pipe is balanced by the Coulomb repulsion from the diffusing photoelectrons of the neighboring pipes, resulting in such a distorted pattern. This shows that the involved diffusion processes mostly correspond to free charged particles, namely the induced photoelectrons. This frustrated diffusion is also depicted in Fig. 3(b) by plotting the cross sections of the fluorescent patterns of Fig. 3(a) along the dashed line across the diameters of two neighboring pipes, for different pulse energies. This evidences the decrease of the inter distance $\Delta l$ between two close fluorescent walls from two neighboring pipes, depending on the incident laser irradiance. The 3D distribution of the pattern obtained with $E_{p}=52 \mathrm{~nJ}$ has been deconvolved (AutoQuant algorithm), permitting us to estimate the thickness of the fluorescent walls to about $210 \pm$ $30 \mathrm{~nm}$, which appears relevant for photonic applications. The evolution of the inter distance $\Delta l$ versus pulse energy is plotted [Fig. 3(d)]. We observe that the minimal interdistance between two fluorescent walls saturates to a minimal value, corresponding to a plateau behavior around $500 \mathrm{~nm}$ [Fig. 3(d)]. A quantitative assessment of this behavior is beyond the scope of this work, and remains an open issue.

Finally, fluorescence and SHG correlative microscopy has been performed on a charge-2 vortex-induced pattern. Fluorescence shows a nested double-ring pattern [Fig. 4(a), also depicted in Fig. 2(h)] with radial symmetry. The SHG pattern, created by direct laser poling, also shows ring patterns, but with distinct diameters and angular dependence [Fig. 4(b)]. The cross sections along the vertical dashed lines give a better insight into the fluorescence and SHG profiles. The latter profile mostly shows two main concentric rings [Fig. $\underline{4(\mathrm{c})}$ ],
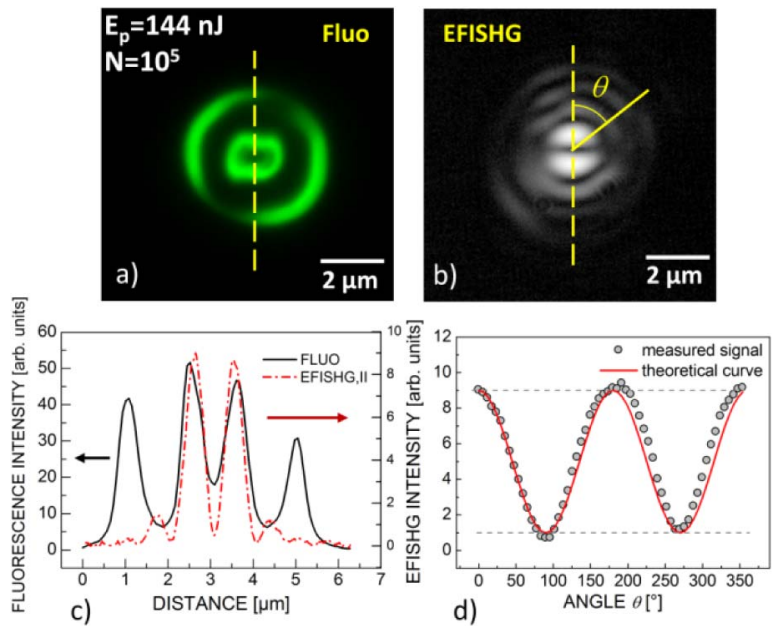

Fig. 4. Fluorescence and SHG correlative microscopy: 2D top views from (a) confocal fluorescence imaging and (b) SHG imaging. (c) Fluorescence and SHG radial profiles along SHG probe beam polarization (dashed line at $\theta=0^{\circ}$ ). (d) Polarization dependence of the EFISHG signal with the typical 9:1 ratio in agreement with the expected angular dependence $9 \cos ^{2}(\theta)+$ $\sin ^{2}(\theta)$. 
the inner ring being more intense than the outer ring. These two rings are located rather close to the edges of the inner fluorescent ring [Fig. 4(c)]. However, the modeling of the relative fluorescence and SHG distributions could not be provided here with vortex-induced DLW, unlike in previous works with Gaussian beams [17]. Still, the incident linearly polarized probe beam (vertical polarization in Fig. 4(b), related to the angle $\theta=0^{\circ}$ ) leads to a radial intensity distribution of the frequencydoubled beam [Fig. 4(d)] with the typical 9:1 ratio between the directions $\theta=0^{\circ}$ and $90^{\circ}$, respectively. This is in excellent agreement with the theoretical model $9 \cos ^{2}(\theta)+\sin ^{2}(\theta)$, proving the radial distribution of the buried electric field and the EFISHG nature of the frequency-doubling nonlinear process [18].

DLW with structured light opens new patterning abilities. First, we notice that the double-wall and four singlewall hollow pipes most probably cannot be obtained by means of successive DLW with a Gaussian beam. Actually, overwriting effects would lead to the erasure by the center of intense spots [14]. Indeed, the edges of the intense spots write fluorescent clusters while their more intense central parts erase them. Second, beyond the complex pattern issue, structured light-induced DLW offers the ability for parallelized optical processing [24], as with the four-lobe beam, by simultaneously writing multiple identical structures. Third, 3D patterns with sub-diffraction features are obtained, since we estimated the thickness of some fluorescence walls down to $210 \pm$ $30 \mathrm{~nm}$ [Fig. 3(c)], which is well below the diffraction limit [14].

More generally, our observations suggest that any structured light, such as Laguerre-Gauss $L_{\mathrm{pl}}$ and Hermite-Gauss $\mathrm{HG}_{\mathrm{nm}}$ light modes, or laser interference patterns, could generate complex photo-induced linear and nonlinear patterns, with periodic, quasi-periodic, or aperiodic features. As an example, $\mathrm{LG}_{\mathrm{pl}}$ beams (corresponding to $p+1$ concentric annular intensity distributions) are expected to produce $2(p+1)$ embedded nested fluorescent rings, while $\mathrm{HG}_{\mathrm{mn}}$ should produce $(n+1) \times(m+1)$ rings. The resulting shaping of the nonlinear optical properties would be worth exploring. Additionally, these nontrivial patterns obtained with structured light could also apply for original spatial modifications of various properties for laser-induced composite materials, such as the local precipitation of metallic silver nanoparticles in these phosphate glasses [25], potentially leading to bulk optical devices with engineered dispersion.

To conclude, in this Letter, we performed original DLW using structured light, which has led to nontrivial fluorescence patterns that cannot be obtained with successive steps of DLW with a Gaussian beam. Moreover, we have discussed the principle of single-beam parallelized DLW by using appropriately shaped light fields. We have also observed sub-diffraction features. Finally, correlative microscopy has confirmed both fluorescent and nonlinear SHG responses, with correlated but distinct spatial distributions of the photo-induced linear and nonlinear optical properties. Such bi-functionality could be exploited for innovative three-dimensional photonic architectures.
This work was funded by French Agency of National Research [FELINS 2010 ANR BLAN 946 03], French Aquitaine Region [FELINS REGION], group of scientific interest AMA LasINOF, Cluster of Excellence LAPHIA, and Photonic Imaging platform of Bordeaux Imaging Center (UMS 420 CNRS).

\section{References}

1. R. R. Gattass and E. Mazur, Nat. Photonics 2, 219 (2008).

2. T. A. Fadeyeva, V. G. Shvedov, Y. V. Izdebskaya, A. V. Volyar, E. Brasselet, D. N. Neshev, A. S. Desyatnikov, W. Krolikowski, and Y. S. Kivshar, Opt. Express 18, 10848 (2010).

3. A. Royon, Y. Petit, G. Papon, M. Richardson, and L. Canioni, Opt. Mat. Express 1, 866 (2011).

4. Y. Shimotsuma, P. G. Kazansky, J. Qiu, and K. Hirao, Phys. Rev. Lett. 91, 247405 (2003).

5. C. Hnatovsky, V. G. Shvedov, W. Krolikowski, and A. V. Rode, Opt. Lett. 35, 3417 (2010).

6. C. Hnatovsky, V. G. Shvedov, N. Shostka, A. V. Rode, and W. Krolikowski, Opt. Lett. 37, 226 (2012).

7. C. Hnatovsky, V. Shvedov, W. Krolikowski, and A. V. Rode, Phys. Rev. Lett. 106, 123901 (2011).

8. S. W. Hell and J. Wichmann, Opt. Lett. 19, 780 (1994).

9. J. Fischer and M. Wegener, Opt. Mat. Express 1, 614 (2011)

10. R. Wollhofen, J. Katzmann, C. Hrelescu, J. Jacak, and T. A. Klar, Opt. Express 21, 10831 (2013).

11. M. Beresna, M. Gecevicius, P. G. Kazansky, and T. Gertus, App. Phys. Lett. 98, 201101 (2011).

12. E. Brasselet, A. Royon, and L. Canioni, Appl. Phys. Lett. 100, 181901 (2012)

13. M. Vangheluwe, F. Liang, Y. Petit, P. Hée, Y. Ledemi, S. Thomas, E. Fargin, T. Cardinal, Y. Messaddeq, L. Canioni, and R. Vallée, Opt. Lett. 39, 5491 (2014).

14. M. Bellec, A. Royon, B. Bousquet, K. Bourhis, M. Treguer, T. Cardinal, M. Richardson, and L. Canioni, Opt. Express 17, 10304 (2009).

15. M. Bellec, A. Royon, K. Bourhis, J. Choi, B. Bousquet, M. Treguer, T. Cardinal, J.-J. Videau, M. Richardson, and L. Canioni, J. Phys. Chem. C 114, 15584 (2010).

16. J. Choi, M. Bellec, A. Royon, K. Bourhis, G. Papon, T. Cardinal, L. Canioni, and M. Richardson, Opt. Lett. 37, 1029 (2012).

17. G. Papon, Y. Petit, N. Marquestaut, A. Royon, M. Dussauze, V. Rodriguez, T. Cardinal, and L. Canioni, Opt. Mat. Express 3, 1855 (2013).

18. G. Papon, N. Marquestaut, Y. Petit, A. Royon, M. Dussauze, V. Rodriguez, T. Cardinal, and L. Canioni, J. Appl. Phys. 115, 113103 (2014).

19. L. Canioni, M. Bellec, A. Royon, B. Bousquet, and T. Cardinal, Opt. Lett. 33, 360 (2008).

20. K. Bourhis, A. Royon, M. Bellec, J. Choi, A. Fargues, M. Treguer, J.-J. Videau, D. Talaga, M. Richardson, T. Cardinal, and L. Canioni, J. Non-Cryst. Solids 356, 2658 (2010).

21. A. V. Volyar and T. A. Fadeeva, Opt. Spectrosc. 94, 235 (2003).

22. Y. Izdebskaya, E. Brasselet, V. Shvedov, A. Desyatnikov, W. Krolikowski, and Y. Kivshar, Opt. Express 17, 18196 (2009).

23. E. Gamaly, S. Juodkazis, V. Mizeikis, H. Misawa, A. V. Rode, and W. Krolikowski, Phys. Rev. B 81, 054113 (2010).

24. P. Bingen, M. Reuss, J. Engelhardt, and S. W. Hell, Opt. Express 19, 23716 (2011).

25. N. Marquestaut, Y. Petit, A. Royon, T. Cardinal, and L. Canioni, Adv. Funct. Mat. 24, 5824 (2014). 